\title{
Estimates of Solutions During Motion of the Euler - Poinsot Top and Explanation of the Experiment with Dzhanibekov's Nut
}

\author{
V.F. Zhuravlev, G. M. Rozenblat
}

This paper presents secure upper and lower estimates for solutions to the equations of rigid body motion in the Euler case (in the absence of external torques). These estimates are expressed by simple formulae in terms of elementary functions and are used for solutions that are obtained in a neighborhood of the unstable steady rotation of the body about its middle axis of inertia. The estimates obtained are applied for a rigorous explanation of the flip-over phenomenon which arises in the experiment with Dzhanibekov's nut.

Keywords: Euler top, permanent (steady) rotation, middle axis of inertia, estimates of solutions to differential equations

\section{Introduction}

As is well known [1, 2], the solution to the Euler - Poinsot problem (a heavy rigid body with a point fastened at its center of gravity) is expressed for almost any initial conditions in terms of elliptic functions. An exception are some initial conditions (of zero measure in phase space) for which solutions are asymptotic (aperiodic) and are expressed in terms of hyperbolic functions. The problem of interest is that of obtaining secure bilateral (upper and lower) estimates of all these solutions, which are expressed by simple formulae in terms of elementary functions.

Received November 26, 2019

Accepted August 24, 2020

Viktor F. Zhuravlev

zhurav@ipmnet.ru

Institute for Problems in Mechanics of the Russian Academy of Sciences

prosp. Vernadskogo 101, Moscow, 119526 Russia

Grigory M. Rozenblat

gr51@mail.ru

Moscow Automobile and Road Construction State Technical University

Leningradsky prosp. 64, Moscow, 125319 Russia 
These estimates turn out to be particularly useful and practical for solutions that are obtained in a neighborhood of unstable steady rotations of the body about its middle axis of inertia. In this paper, such estimates are obtained for the nutation angle $\theta(t)$ (more precisely, for the cosine of this angle), which is, by definition, the angle between the middle axis of inertia of the body and the body's angular momentum vector (fixed in inertial space) relative to its center of gravity (for the Euler-Poinsot case). Similar estimates can also be obtained for other Euler angles which describe the motion of the rigid body in space.

In particular, the estimates obtained for the cosine of the nutation angle allow a mathematically rigorous and quantitative explanation of the essence of the so-called experiment with Dzhanibekov's nut, which was previously qualitatively considered in [3, 4] using approximate (asymptotic) methods. Ultimately, it follows from this that the motion of Dzhanibekov's nut is not by any means a new phenomenon in science, but nothing more than a special case of rigid body motion (the Euler-Poinsot case), which was obtained and described by the great L. Euler over three hundred years ago.

We note that analogous estimates of solutions were obtained earlier for the Lagrange top in $[5,6]$ (for estimation of the middle angular velocity of precession in the Hadamard problem $[7,8]$ ) and for a gyroscope in a Cardan suspension in [9] (for explanation of the so-called Magnus effect).

\section{Notation and the main equations of the problem}

Let $O$ be the center of gravity of the body and let $O \xi \eta \zeta$ be a moving right-handed coordinate system rigidly attached to the body with axes directed along the principal axes of inertia of the body for point $O$. Let $A, B$ and $C$ denote the principal moments of inertia of the body relative to the axes $O \xi, O \eta$ and $O \zeta$, respectively. Further, we assume that $A>B>C$. Thus, $O \eta$ is the middle axis of inertia of the body.

Let $O x y z$ be the inertial right-handed coordinate system with axis $O z$ directed along the constant (in inertial space) angular momentum vector $K$ of the body relative to its center of gravity $O, \theta$ the angle between the axes $O \eta$ and $O z$ (see the Introduction), and $\omega$ the angular velocity vector of the body.

Let us introduce symbols $X, Y$ and $Z$ for the respective projections of the angular momentum vector $K$ onto the axes of the moving coordinate system $O \xi \eta \zeta$ using the formulae

$$
X=K_{\xi}=A \omega_{\xi}, \quad Y=K_{\eta}=B \omega_{\eta}, \quad Z=K_{\zeta}=C \omega_{\zeta}
$$

Then we obtain $\cos \theta=Y / K$, where $K$ is the absolute value of the constant angular momentum vector of the body. In this case, we have two classical integrals (constancy of the absolute value of the angular momentum and constancy of the kinetic energy of the body):

$$
X^{2}+Y^{2}+Z^{2}=K^{2}=\text { const }, \quad \frac{X^{2}}{A}+\frac{Y^{2}}{B}+\frac{Z^{2}}{C}=2 T=\text { const. }
$$

To completely solve the problem, one of three dynamical Euler equations should be added to Eqs. (1.1). If we are interested in the time behavior of the function $Y(t)$ and consequently of the function $\theta(t)=\arccos [Y(t) / K]$, then it is convenient to take the following Euler equation:

$$
\frac{d Y}{d t}=-(A-C) \frac{X Z}{A C}
$$


From (1.1) and the dynamical Euler equations it follows that the steady motion about the middle axis of inertia $O \eta$ corresponds to initial conditions satisfying the relation $Y^{2}(0)=K^{2}=2 T B$. If $Y^{2}(0) \neq K^{2}(|Y(0)|<K$, for example, if $Y(0)=0, \theta(0)=\pi / 2)$, but the condition $K^{2}=2 T B$ is fulfilled, then, as is well known, asymptotic (aperiodic) body motion occurs at $t \in[0, \infty)$. This motion can be thought of (in the moving coordinate system $O \xi \eta \zeta$ attached to the rigid body) as a monotonous evolution of the body's angular momentum vector $K$ to the positive or negative direction of its middle axis of inertia $O \eta$. These motions of the vector $K$ occur asymptotically, at $t \in[0, \infty)$, in the above-mentioned moving coordinate system $O \xi \eta \zeta$ along separatrices on MacCullagh's ellipsoid. This ellipsoid is given by the second of relations (1.1) (for a geometric interpretation of MacCullagh's motion, see, e.g., [10-12]). The separatrices are formed as a result of intersections of MacCullagh's ellipsoid with the sphere in relations (1.1), subject to the condition $K^{2}=2 T B$.

Next, we will study the body's motions in some neighborhood of the separatrix. To do so, we introduce the parameter $\varepsilon=K^{2}-2 T B \neq 0$, which is, generally speaking, a small quantity, although this is not assumed in the main results presented below. Below, without loss of generality, we assume that $\varepsilon>0$.

We introduce the positive parameters

$$
\begin{aligned}
\lambda_{0}=\frac{A C}{B(A-C)}, & \lambda_{1}=\frac{A(B-C)}{B(A-C)}, \\
\lambda_{2}=\frac{C(A-B)}{B(A-C)}, & \lambda_{3}=\frac{A-C}{A C} \sqrt{\lambda_{1} \lambda_{2}} .
\end{aligned}
$$

We note that the parameters $\lambda_{k}(k=0,1,2)$ in (1.3) are dimensionless and the parameter $\lambda_{3}$ has a dimension inverse to that of the moment of inertia. Then from (1.1) we have

$$
X^{2}=\lambda_{1}\left(K^{2}-Y^{2}\right)+\lambda_{0} \varepsilon, \quad Z^{2}=\lambda_{2}\left(K^{2}-Y^{2}\right)-\lambda_{0} \varepsilon, \quad \varepsilon=K^{2}-2 T B>0 .
$$

Substituting $X$ and $Z$ from (1.4) into (1.2), we obtain a differential equation with separating variables

$$
\frac{d Y}{d t}= \pm \lambda_{3} \sqrt{\left(Y_{1}^{2}-Y^{2}\right)\left(Y_{2}^{2}-Y^{2}\right)}
$$

In (1.5) the parameter $\lambda_{3}$ is defined by (1.3) and the following notation is introduced:

$$
Y_{1}^{2}=K^{2}+\frac{\lambda_{0} \varepsilon}{\lambda_{1}}>0, \quad Y_{2}^{2}=K^{2}-\frac{\lambda_{0} \varepsilon}{\lambda_{2}}>0 \quad \text { for } 0<\varepsilon<\frac{\lambda_{2} K^{2}}{\lambda_{0}}
$$

In (1.6) the parameters $\lambda_{0}, \lambda_{1}, \lambda_{2}, \varepsilon$ are defined by (1.3) and (1.4). The system (1.6) contains an inequality that is satisfied by the deviation value of $\varepsilon$. As we see, this inequality does not imply smallness of $\varepsilon$.

Analysis of Eq. (1.5) shows that the function $Y(t)$ is $T$-periodic and changes in the interval $\left[-Y_{2}, Y_{2}\right]$. Then the initial condition $Y(0)$ also satisfies the inequality $|Y(0)|<Y_{2}$. The period of the function $Y(t)$ is given by the formula

$$
T=\frac{2}{\lambda_{3}} \int_{-Y_{2}}^{Y_{2}} \frac{d Y}{\sqrt{\left(Y_{1}^{2}-Y^{2}\right)\left(Y_{2}^{2}-Y^{2}\right)}}=\frac{4}{\lambda_{3}} \int_{0}^{Y_{2}} \frac{d Y}{\sqrt{\left(Y_{1}^{2}-Y^{2}\right)\left(Y_{2}^{2}-Y^{2}\right)}} .
$$

The integral in (1.7) is a complete elliptic integral of the first kind $[13,14]$.

RUSSIAN JOURNAL OF NONLINEAR DYNAMICS, 2020, 16(3), 517-525 


\section{Formulation of the problem}

In the general case, Eq. (1.5) cannot be integrated by elementary functions. This raises the problem of obtaining bilateral (upper and lower) secure estimates of solutions to Eq. (1.5) subject to conditions (1.6). It is also necessary to obtain analogous bilateral estimates for period $T$ from (1.7). These estimates must be sufficiently simple for practical use and expressed in terms of elementary functions. In addition, in the limit as $\varepsilon \rightarrow 0$, they must give an exact solution to the problem, i.e., an asymptotic motion along a separatrix as described above (see [10-12]).

\section{Formulation and proof of results}

We integrate Eq. (1.5), assuming that the right-hand side has the sign + and $Y(0)=0$ (i.e., the initial value of the nutation angle is $\theta(0)=\pi / 2$ ):

$$
\lambda_{3} t=\int_{0}^{Y} \frac{d Y}{\sqrt{\left(Y_{1}^{2}-Y^{2}\right)\left(Y_{2}^{2}-Y^{2}\right)}}, \quad Y \in\left[0, Y_{2}\right] .
$$

We now introduce the notation

$$
\begin{gathered}
z_{1}(t)=\frac{1}{Y_{1}+Y(t)}, \quad z_{2}(t)=\frac{1}{Y_{2}+Y(t)}, \\
s_{1}=\sqrt{\frac{Y_{1}\left(Y_{1}+Y_{2}\right)}{2}}, \quad s_{2}=\sqrt{\frac{Y_{2}\left(Y_{1}+Y_{2}\right)}{2}}, \\
\sigma_{1}=\frac{\sqrt{Y_{1}+Y_{2}}+\sqrt{2 Y_{2}}}{\sqrt{2 Y_{1}\left(Y_{1}+Y_{2}\right)}}, \quad \sigma_{2}=\frac{\sqrt{Y_{1}+Y_{2}}+\sqrt{2 Y_{1}}}{\sqrt{2 Y_{2}\left(Y_{1}+Y_{2}\right)}}, \\
a=\frac{1}{Y_{1}+Y_{2}}, \quad b_{1}=\frac{1}{2 Y_{1}}, \quad b_{2}=\frac{1}{2 Y_{2}} .
\end{gathered}
$$

The following theorem holds.

Theorem. For the functions $z_{1}(t), z_{2}(t)$ expressed using the formulae from (3.2) in terms of the solution $Y(t)$ to equation (3.1) on the interval $t \in(0, T)$, the following inequalities hold:

$$
\frac{1}{s_{1}} \ln \left(\frac{\sigma_{1}}{\sqrt{z_{1}(t)-a}+\sqrt{z_{1}(t)-b}}\right) \leqslant \lambda_{3} t \leqslant \frac{1}{s_{2}} \ln \left(\frac{\sigma_{2}}{\sqrt{z_{2}(t)-a}+\sqrt{z_{2}(t)-b}}\right) .
$$

In (3.3) all parameters are defined by (3.2) and (1.3).

Proof The proof of the theorem follows from the bilateral estimates of the integral on the right-hand side of Eq. (3.1):

$$
\begin{aligned}
\int_{0}^{Y} \frac{d Y}{\left(Y_{1}+Y\right) \sqrt{\left(Y_{1}-Y\right)\left(Y_{2}-Y\right)}}<\int_{0}^{Y} \frac{d Y}{\sqrt{\left(Y_{1}^{2}-Y^{2}\right)\left(Y_{2}^{2}-Y^{2}\right)}}< \\
\quad<\int_{0}^{Y} \frac{d Y}{\left(Y_{2}+Y\right) \sqrt{\left(Y_{1}-Y\right)\left(Y_{2}-Y\right)}}
\end{aligned}
$$


the validity of which follows from the inequality $Y_{1}>Y_{2}$ with $\varepsilon>0$ (see (1.6)). The last integrals in inequalities (3.4) are taken in elementary functions:

$$
\begin{aligned}
\int \frac{d Y}{\left(Y_{1}+Y\right) \sqrt{\left(Y_{1}-Y\right)\left(Y_{2}-Y\right)}} & =-\frac{1}{\sqrt{2 Y_{1}\left(Y_{1}+Y_{2}\right)}} \int \frac{d z_{1}}{\sqrt{\left(z_{1}-a\right)\left(z_{1}-b\right)}}= \\
& =-\frac{1}{s_{1}} \ln \left(\sqrt{z_{1}-a}+\sqrt{z_{1}-b}\right) \\
\int \frac{d Y}{\left(Y_{2}+Y\right) \sqrt{\left(Y_{1}-Y\right)\left(Y_{2}-Y\right)}} & =-\frac{1}{\sqrt{2 Y_{2}\left(Y_{1}+Y_{2}\right)}} \int \frac{d z_{2}}{\sqrt{\left(z_{2}-a\right)\left(z_{2}-b\right)}}= \\
& =-\frac{1}{s_{2}} \ln \left(\sqrt{z_{2}-a}+\sqrt{z_{2}-b}\right)
\end{aligned}
$$

where $z_{1}, z_{2}, a, b_{1}, b_{2}, s_{1}, s_{2}$ are defined by (3.2). Substituting the resulting expressions into inequalities (3.4) and using the Newton-Leibniz formula for calculating the defined integrals, we obtain relations (3.3) of the theorem.

This proves the theorem.

Corollary 1. Denote $t_{*}=T / 4$, where $T$ is the full period of the solution as defined by formula (1.7). Then the following estimates hold:

$$
\begin{gathered}
t_{1}<t_{*}<t_{2}, \\
t_{1}=\frac{1}{\lambda_{3} s_{1}} \ln \left(\frac{\sqrt{2 Y_{2}}+\sqrt{Y_{1}+Y_{2}}}{\sqrt{Y_{1}-Y_{2}}}\right), \\
t_{2}=\frac{1}{\lambda_{3} s_{2}} \ln \left(\frac{\sqrt{2 Y_{1}}+\sqrt{Y_{1}+Y_{2}}}{\sqrt{Y_{1}-Y_{2}}}\right) .
\end{gathered}
$$

Proof of Corollary 1. Inequalities (3.5) follow immediately from inequalities (3.3) of the theorem when $t=t_{*}$ and $Y\left(t_{*}\right)=Y_{2}$ are substituted into (3.3).

Corollary 2. Inequalities (3.3) are equivalent to the following inequalities:

$$
\begin{aligned}
z_{1}(t) \geqslant z_{11}(t)= & \frac{1}{4} \sigma_{1}^{2} e^{-2 \lambda_{3} s_{1} t}+\frac{1}{2}\left(\frac{1}{Y_{1}+Y_{2}}+\frac{1}{2 Y_{1}}\right)+ \\
& +\frac{1}{4 \sigma_{1}^{2}} e^{2 \lambda_{3} s_{1} t}\left[\frac{Y_{1}-Y_{2}}{2 Y_{1}\left(Y_{1}+Y_{2}\right)}\right]^{2}, \quad 0 \leqslant t \leqslant t_{1}, \\
z_{1}(t) \geqslant \frac{1}{Y_{1}+Y_{2}}, & t_{1} \leqslant t \leqslant t_{*}, \\
z_{2}(t) \leqslant z_{21}(t)= & \frac{1}{4} \sigma_{2}^{2} e^{-2 \lambda_{3} s_{2} t}+\frac{1}{2}\left(\frac{1}{Y_{1}+Y_{2}}+\frac{1}{2 Y_{2}}\right)+ \\
& +\frac{1}{4 \sigma_{2}^{2}} e^{2 \lambda_{3} s_{2} t}\left[\frac{Y_{1}-Y_{2}}{2 Y_{2}\left(Y_{1}+Y_{2}\right)}\right]^{2}, \quad t \in\left[0, t_{*}\right] .
\end{aligned}
$$

Remarks to Corollary 2.

1. It is easy to show that at $t=0$ inequalities (3.6) turn into equalities, with $z_{1}(0)=$ $=z_{11}(0)=1 / Y_{1}$ and $z_{2}(0)=z_{21}(0)=1 / Y_{2}$. 
2. Using inequalities (3.6) and the notation from (3.2) for the functions $z_{1}(t)$ and $z_{2}(t)$, it is easy to obtain corresponding lower and upper estimates for the solution $Y(t)$ itself (and for the nutation angle $\theta(t)=\arccos [Y(t) / K])$ :

$$
\begin{array}{ll}
-Y_{2}+\frac{1}{z_{21}(t)} \leqslant Y(t) \leqslant-Y_{1}+\frac{1}{z_{11}(t)}, & t \in\left[0, t_{1}\right), \\
-Y_{2}+\frac{1}{z_{21}(t)} \leqslant Y(t) \leqslant Y_{2}, & t \in\left[t_{1}, t_{*}\right] .
\end{array}
$$

In (3.7) the functions $z_{11}(t), z_{21}(t)$ are defined by (3.6). Here too, at $t=0$ inequalities (3.7) turn into equalities. Relations (3.7) show that the solution $Y(t)$ remains for the entire period in some "tube" whose boundaries are defined by (3.7) and expressed by simple formulae in terms of elementary functions.

Proof of Corollary 2. Rewrite inequalities (3.3) of the theorem as

$$
\begin{aligned}
& \sqrt{z_{1}(t)-a}+\sqrt{z_{1}(t)-b} \geqslant \sigma_{1} e^{-\lambda_{3} s_{1} t}, \\
& \sqrt{z_{2}(t)-a}+\sqrt{z_{2}(t)-b} \leqslant \sigma_{2} e^{-\lambda_{3} s_{2} t} .
\end{aligned}
$$

Inequalities (3.8) hold in the following domains of definitions:

$$
z_{1} \geqslant a=\frac{1}{Y_{1}+Y_{2}}, \quad z_{2} \geqslant b_{2}=\frac{1}{2 Y_{2}}
$$

since, by virtue of the notation of $(3.2), b_{2} \geqslant a \geqslant b_{1}$ when $0 \leqslant Y_{2} \leqslant Y_{1}$.

Since the left-hand sides of inequalities (3.8) are monotonous in $z_{1}$ and $z_{2}$, the functions $z_{1}(t)$ and $z_{2}(t)$ in (3.8) are bounded, respectively, from below and above by the roots of the equations

$$
\begin{aligned}
& \sqrt{z_{1}(t)-a}+\sqrt{z_{1}(t)-b}=\sigma_{1} e^{-\lambda_{3} s_{1} t}, \\
& \sqrt{z_{2}(t)-a}+\sqrt{z_{2}(t)-b}=\sigma_{2} e^{-\lambda_{3} s_{2} t} .
\end{aligned}
$$

A straightforward calculation shows that the functions $z_{11}(t)$ and $z_{21}(t)$ which are defined in (3.6) are exactly the roots of Eqs. (3.9). Also, the following circumstance needs to be taken into account. These roots exist only at those $t$ for which the following inequalities are satisfied:

$$
\sigma_{1} e^{-\lambda_{3} s_{1} t} \geqslant \sqrt{a-b_{1}}, \quad \sigma_{2} e^{-\lambda_{3} s_{2} t} \geqslant \sqrt{b_{2}-a} .
$$

Using the notation of (3.2), it is easy to show that the first of inequalities (3.10) is satisfied for $0 \leqslant t \leqslant t_{1}$, and the second, for $0 \leqslant t \leqslant t_{2}$, where $t_{1}$ and $t_{2}$ are defined by (3.5). Thus, for $z_{1}(t)$ we obtain the estimates

$$
z_{1}(t) \geqslant z_{11}(t), \quad 0 \leqslant t \leqslant t_{1}, \quad z_{1}(t) \geqslant \frac{1}{Y_{1}+Y_{2}}, \quad t_{1}<t \leqslant t_{*} .
$$

And since, by virtue of Corollary 1 , the inequality $t_{*}<t_{2}$ holds, it follows that for $z_{2}(t)$ the estimate $z_{2}(t) \leqslant z_{21}(t), 0 \leqslant t \leqslant t_{*}$ holds. This yields inequalities (3.6), and Corollary 2 is proved. 
Corollary 3. If $\varepsilon \rightarrow 0$, then inequalities (3.3) of the theorem turn into equalities, and the resulting solution $Y(t)$ turns into the well-known classical asymptotic solution according to the law of hyperbolic tangent.

Proof of Corollary 3. For $\varepsilon=0$, from (1.6) we have $Y_{1}=Y_{2}=K$, and from (3.2), (3.3) we obtain

$$
z_{1}(t)=z_{2}(t)=\frac{1}{K+Y(t)}, \quad Y(t)=K \tanh (\alpha t), \quad \alpha=K \lambda_{3} .
$$

The validity of the last formulae is established by straightforward verification. Corollary 3 is proved.

Corollary 4 (explanation of the experiment with Dzhanibekov's nut). Using estimates (3.6) or (3.7) of Corollary 2 for the solution $Y(t)$ and the nutation angle $\theta(t)$, one can show that for any $\delta>0$ and for sufficiently small $\varepsilon>0$ the solution $Y(t)$ is in the $\delta$ neighborhood of the point $Y=Y_{2}(\theta(t) \approx 0)$ or of the point $Y=-Y_{2}(\theta(t) \approx \pi)$ for a long time of order $|\ln \varepsilon|$. In contrast, the transition from the small neighborhood of the point $Y= \pm Y_{2}$ (where $\theta(t) \approx 0$ or $\theta(t) \approx \pi$ ) to the small neighborhood of the point $Y=\mp Y_{2}$ (where $\theta(t) \approx \pi$ or $\theta(t) \approx 0$ ) lasts for a finite time, which, in zero approximation (as $\varepsilon \rightarrow 0)$, does not depend on $\varepsilon$, but, of course, does depend on $\delta$.

Proof of Corollary 4 follows from the fact that the upper and lower estimates of the solution $Y(t)$, which follow from inequalities (3.7) of Corollary 2, approximate uniformly in $t \in\left[0, t_{*}\right]$, as $\varepsilon \rightarrow 0$, the hyperbolic tangent of the solution from Corollary 3 .

We present a more rigorous and constructive proof of Corollary 4, which is intended for explanation of the well-known experiment with Dzhanibekov's nut. In this experiment, which was carried out in outer space (under weightlessness conditions) by the space pilot V. A. Dzhanibekov, twice Hero of the Soviet Union, the mounting wing nut was set in rectilinear motion with fast initial rotation about the axis close to the middle principal axis of inertia of the wing nut (with its wing-like projections directed forward, in the direction of its initial straight-line motion). After a fairly long and almost steady rotation about this axis the nut suddenly flipped over rapidly by 180 degrees and continued again a fairly long rotation (with its wing-like projections directed backward, i.e., against its initial rectilinear motion). In Dzhanibekov's experiment described above, these flip-overs were observed strictly periodically. As can be seen, the above experiment is a practical application of the solution to the problem of the motion of the Euler - Poinsot top, which is addressed in this paper.

For a quantitative proof of the above-mentioned experiment with Dzhanibekov's nut we proceed as follows. We give the following physical meanings to the parameters $\delta$ and $\varepsilon$ which appear in the formulation of Corollary 4 . We call $\delta$ the threshold (sensitivity threshold). This threshold is the extreme deviation of the middle axis of inertia of the nut from the direction of the angular momentum vector $K$ (constant in inertial space), i.e., the deviation of the angle $\theta(t)$ from the values $\theta=0$ or $\theta=\pi$ ), which we (more precisely, our eyes) assume to be sufficiently small (imperceptible) in Dzhanibekov's experiment. We will call the parameter $\varepsilon=K^{2}-2 T B$ adjustment. The adjustment characterizes the accuracy with which we set the nut in motion in the experiment at the initial instant of time so as to satisfy the equation $K^{2}=2 T B$ as well as possible. Then, as stated above, the nut will move in a small neighborhood of the separatrix. It is clear that the value $\varepsilon=0$ cannot be attained in this experiment.

For a convincing and effective demonstration in Dzhanibekov's experiment described above, it is necessary to choose the parameter $\varepsilon$ (adjustment) to be sufficiently small, in contrast 
to the parameter $\delta$ (sensitivity threshold), which is, generally speaking, not very small and may be assumed to be finite and fixed in further reasoning. We rewrite inequalities (3.7) as

$$
\begin{array}{rlrl}
Y_{1}+Y_{2}-\frac{1}{z_{11}(t)} \leqslant Y_{2}-Y(t) \leqslant 2 Y_{2}-\frac{1}{z_{21}(t)}, & & 0 \leqslant t \leqslant t_{1}, \\
0 & \leqslant Y_{2}-Y(t) \leqslant 2 Y_{2}-\frac{1}{z_{21}(t)}, & & t_{1} \leqslant t \leqslant t_{*} .
\end{array}
$$

Using the first formula for $z_{11}(t)$ from relations (3.6) and the Cauchy-Bunyakovsky inequality, it is easy to obtain the inequalities

$$
z_{11}(t) \geqslant \frac{1}{Y_{1}+Y_{2}} \quad \rightarrow \quad Y_{1}+Y_{2}-\frac{1}{z_{11}(t)} \geqslant 0 .
$$

This implies that inequalities (3.11) lead to the inequalities

$$
0 \leqslant Y_{2}-Y(t) \leqslant 2 Y_{2}-\frac{1}{z_{21}(t)}, \quad 0 \leqslant t \leqslant t_{*} .
$$

Thus, the deviation of the solution $Y(t)$ from $Y_{2}$ is determined by the behavior of the function $z_{21}(t)$ from (3.6). The function $z_{21}(t)$, which is defined by (3.6), consists of three terms. For the third term the following estimate holds:

$$
\frac{1}{4 \sigma_{2}^{2}} e^{2 \lambda_{3} s_{2} t}\left[\frac{Y_{1}-Y_{2}}{2 Y_{2}\left(Y_{1}+Y_{2}\right)}\right]^{2}<\frac{Y_{1}-Y_{2}}{8 Y_{1}\left(Y_{1}+Y_{2}\right)} \leqslant C_{2} \varepsilon, \quad t \in\left[0, t_{*}\right]
$$

where $C_{2}=$ const, which does not depend on $\varepsilon$. Thus, the function $z_{21}(t)$ (as follows from its form) is for sufficiently small $\varepsilon$ monotonically decreasing in $t$ over the whole interval $\left[0, t_{*}\right]$. Therefore, we choose time $t_{3}$ as the root of the equation

$$
2 Y_{2}-\frac{1}{z_{21}(t)}=\delta
$$

Then, in accordance with inequalities (3.12) and monotonic decrease in the function $z_{21}(t)$ over the whole interval $\left[0, t_{*}\right]$, it can be asserted that for sufficiently small $\varepsilon$, in a time not greater than $t_{3}$, the middle axis of inertia of the body will find itself in the $\delta$-neighborhood of the angular momentum $K$ constant in space and will stay there at least until time $t=t_{*}<t_{2}$, i.e., over the whole time interval $\left[t_{3}, t_{*}\right]$. It only remains to note that, as $\varepsilon \rightarrow+0$, time $t_{3}$, being the root of equation (3.13), remains finite and tends to the value $t_{30}=\frac{1}{2 \lambda_{3} K} \ln \frac{2 K-\delta}{\delta}$, i.e., it depends only on $\delta$, and the time interval $\left(t_{*}-t_{3}\right)$ tends, in accordance with the formulae from (3.5), to infinity as $|\ln \varepsilon|$. The above reasoning clearly explains the seemingly strange behavior of the rigid body (Dzhanibekov's nut) in Dzhanibekov's experiment described in the media.

We note that the results obtained here also explain the "strange" behavior of a tennis racket as described in [15].

\section{References}

[1] Arkhangel'skii, Yu. A., Analytical Dynamics of a Rigid Body, Moscow: Nauka, 1977 (Russian).

[2] Appel, P., Traité de Mécanique rationnelle: Vol.2. Dynamique des systèmes. Mécanique analytique, 6th ed., Paris: Gauthier-Villars, 1953. 
[3] Petrov, A. G. and Volodin, S. E., Janibekov's Effect and the Laws of Mechanics, Dokl. Phys., 2013, vol. 58, no. 8, pp. 349-353; see also: Dokl. Akad. Nauk, 2013, vol.451, no.4, pp. 399-403.

[4] Zhuravlev, V.F., Petrov, A. G., and Shunderyuk, M. M., Selected Problems of Hamiltonian Mechanics, Moscow: Lenand, 2015 (Russian).

[5] Rozenblat, G. M., Estimates of the Average Angular Velocity of the Precession of Lagrange's Top, Dokl. Phys., 2019, vol.64, no.3, pp.114-119; see also: Dokl. Akad. Nauk, 2019, vol.485, no. 2, pp. 176-181.

[6] Rozenblat, G. M., Gyroscopic Effects in Mechanics of Solids, 2nd ed., Moscow: Lenand, 2020 (Russian).

[7] Hadamard, J., Sur la précession dans le mouvement d'un corps pesant de révolution fixé par un point de son axe, Bull. Sci. Math., 1895, vol. 19, pp. 228-230.

[8] MacMillan, W. D., Dynamics of Rigid Bodies, New York: McGraw-Hill, 1936.

[9] Zhuravlev, V.F., On the Question of Estimates of the Magnus Effect, Dokl. Akad. Nauk SSSR, 1976, vol. 226, no. 3, pp. 541-543 (Russian).

[10] Zhuravlev, V.F., Foundations of Theoretical Mechanics, 3rd ed., Moscow: Fizmatlit, 2008 (Russian).

[11] Borisov, A. V. and Mamaev, I. S., Rigid Body Dynamics: Hamiltonian Methods, Integrability, Chaos, Izhevsk: R\&C Dynamics, Institute of Computer Science, 2005 (Russian).

[12] Magnus, K., Kreisel: Theorie und Anwendungen, Berlin: Springer, 1971.

[13] Byrd, P. F. and Friedman, M. D., Handbook of Elliptic Integrals for Engineers and Scientists, 2nd ed., rev., Grundlehren Math. Wiss., vol.67, Heidelberg: Springer, 1971.

[14] Erdélyi, A., Magnus, W., Oberhettinger, F., and Tricomi, F. G., Higher Transcendental Functions: Vol. 3. Based, in Part, on Notes Left by Harry Bateman, New York: McGraw-Hill, 1955.

[15] Ashbaugh, M.S., Chicone, C.C., and Cushman, R.H., The Twisting Tennis Racket, J. Dynam. Differential Equations, 1991, vol. 3, no. 1, pp. 67-85. 